\title{
Cutaneous Rosai - Dorfman disease: a case report of disease limited only to skin
}

A. Kontrimiene ${ }^{1}$ E. Serpytiene ${ }^{1}$, M. Macejevska ${ }^{1}$, J. Lauraitis $^{1}$, J. Grigaitiene ${ }^{1}$

${ }^{1}$ Vilnius University Hospital Santariskiu Klinikos, Dermatovenereology department, Vilnius, 08661

Corresponding author: A. Kontrimiene, J. Kairiūkščio g. 2, LT-08411, Vilnius, Lithuania, +37067846045, aurejabareikyte@gmail.com

Key words: Rosai-Dorfman disease, Cutaneous, Case report

Authors ${ }^{6}$ contributions: Aureja Kontrimiene had the conception of the work. Aureja Kontrimiene and Emilija Serpytiene collected the data. Data analysis and interpretation were performed by Aureja Kontrimiene, Emilja Serpytiene, Monika Macejevska. Aureja Kontrimiene, Emilija Serpytiene, Monika Macejevska drafted the article. Jurate Grigaitiene and Jonas Lauraitis performed critical revision of the article. Final approval of the version to be published was given by Emilija Serpytiene, Monika Macejevska, Jonas Lauraitis, Jurate Grigaitiene.

The authors declare no conflict of interest.

This case was presented in $27^{\text {th }}$ EADV Congress in Paris, France, in 2018. 


\begin{abstract}
Rosai - Dorfman disease is a disorder of histiocytic proliferation that can affect almost every organ. Skin may be involved in about $10 \%$ cases, but only $3 \%$ of patients have skin lesions exclusively. Patients with CRDD usually are healthy, without leading constitutional or other symptoms. Etiology of this disease is unclear, but it might be triggered by an infectious agent such as Herpesvirus 6 and Epstein-Barr virus, there is also predisposition to autoimmune diseases, hematological malignancies. In our case patient was diagnosed with Borrelia burgdorferi infection. Any known association between this infection and Rosai - Dorfman disease was not described before. The disease may manifest in any age group. The differential diagnosis includes Langerhans cell histiocytosis, metastasis, Hodgkin's lymphoma and other diseases. Disease usually resolves spontaneously over a period of months to years. Treatment should be administered in nonresponsive or multiple relapsing cases. Also, it is very important to take into consideration the patient's expectations and aesthetic appearance before making a treatment decision. Various treatment options are described, including surgical excision, cryotherapy, corticosteroids, antibiotics, Methotrexate, Dapsone. In our case, we used CO2 laser treatment successfully. To our knowledge, this is only the second case where CRDD lesions were treated successfully with CO2 laser.
\end{abstract}

\title{
Introduction
}

Rosai - Dorfman disease is a rare benign disorder of histiocytic proliferation, which is also known as sinus histiocytosis with massive lymphadenopathy first described in 1969. [1] Extranodal involvement may be seen in $43 \%$ of all cases. The disease can affect almost every organ including skin, kidney, gastrointestinal tract, nervous system and others. Skin may be involved in about $10 \%$ cases, but only $3 \%$ of patients have skin lesions exclusively (without nodal involvement). [2] Etiology of this disease is unclear, but it might be triggered by an infectious agent such as Herpesvirus 6 (HHV-6) and Epstein-Barr virus (EBV), there is also predisposition to autoimmune diseases, hematological malignancies [3] [4] [5]. The disease may manifest in any age group, but more often in younger age [6]. The differential diagnosis includes Langerhans cell histiocytosis, Erdheim-Chester disease, melanoma, metastasis, Hodgkin's lymphoma, xanthomas, histoplasmosis, leishmaniasis, sarcoidosis and other diseases [7] [8].

We present a case of cutaneous Rosai - Dorfman disease which manifested in an older patient. 


\section{Case report}

A 69-year-old male presented to our Dermatology Centre with a 5 months history of erythematous nodules on the skin. No pain or itching was reported. Dermatological examination revealed multiple 0.5 to $1.5 \mathrm{~cm}$ purple, firm nodules and papules located on patient's face, legs and buttocks (Figure 1). General physical examination was unremarkable. No lymphadenopathy or any systemic symptoms were observed. He did not have any relevant medical history and was not taking any medications.


Laboratory investigation, including complete blood count, serum electrolytes, renal function, thyroid, liver function test and glucose, were within normal ranges. Conventional chest radiography and abdominal ultrasound were unremarkable. Additional examinations revealed positive IgG and IgM antibody titers against Borrelia burgdorferi. He denied any recent tick bite. The patient was treated with Amoxicillin $500 \mathrm{mg}$ TID for one month. During the treatment the lesions slowly regressed but after discontinuation of the treatment, the regression stopped. 
Computed tomography of head, chest and abdomen had no abnormal findings. Histological examination of the skin lesion showed infiltration in the papillary and reticular dermis, consisting of medium-sized, abundant pale cytoplasm histiocytes and their groups. Phagocytosed lymphocytes (emperipolesis) were visible (Figure 2).

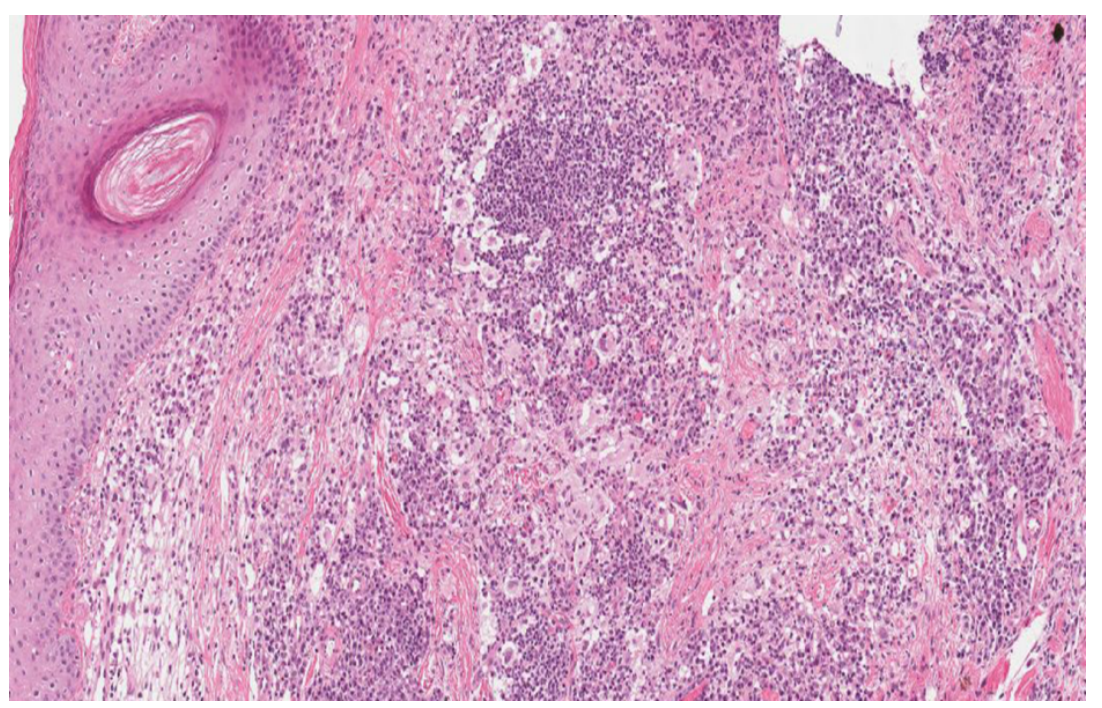

Abundance of lymphocytes and plasma cells were observed in the background. The immunohistochemical examination revealed positive S100 and CD68 staining in histiocytes (Figure $3)$.

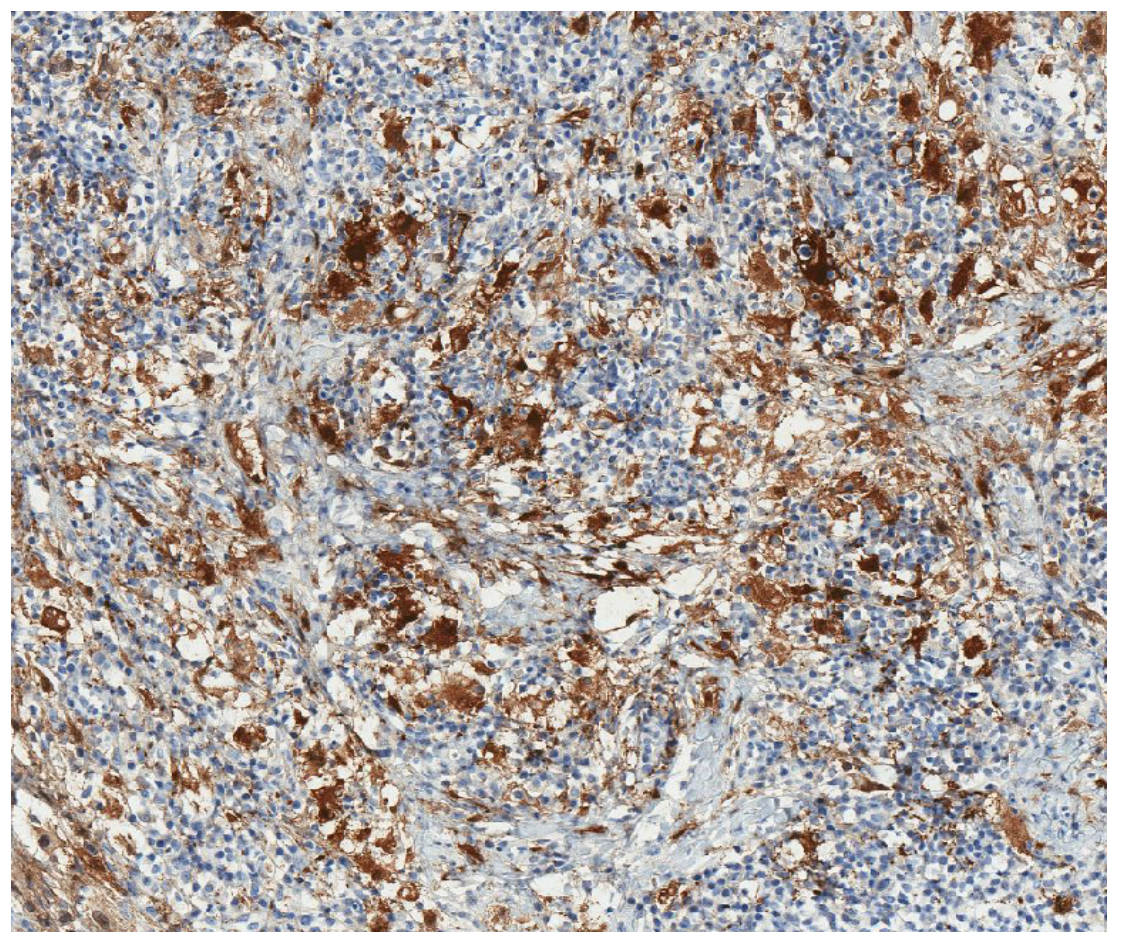

Based on the clinical manifestation of the lesions and histopathological features the diagnosis of cutaneous Rosai - Dorfman disease was established. 
Due to esthetic concerns and slow regression of the lesions, the patient was treated with Dapsone $100 \mathrm{mg}$ BID for two weeks, combined with a Mometasone furoate cream $1 \mathrm{mg} / \mathrm{g}$ QD which showed no effect. So the patient was administered with Methotrexate $20 \mathrm{mg}$ once a week for three months and Triamcinolone acetonide intralesional injections

As an additional treatment option, the patient received four $\mathrm{CO} 2$ laser treatment sessions with a total regression of the lesions (Figure 4).

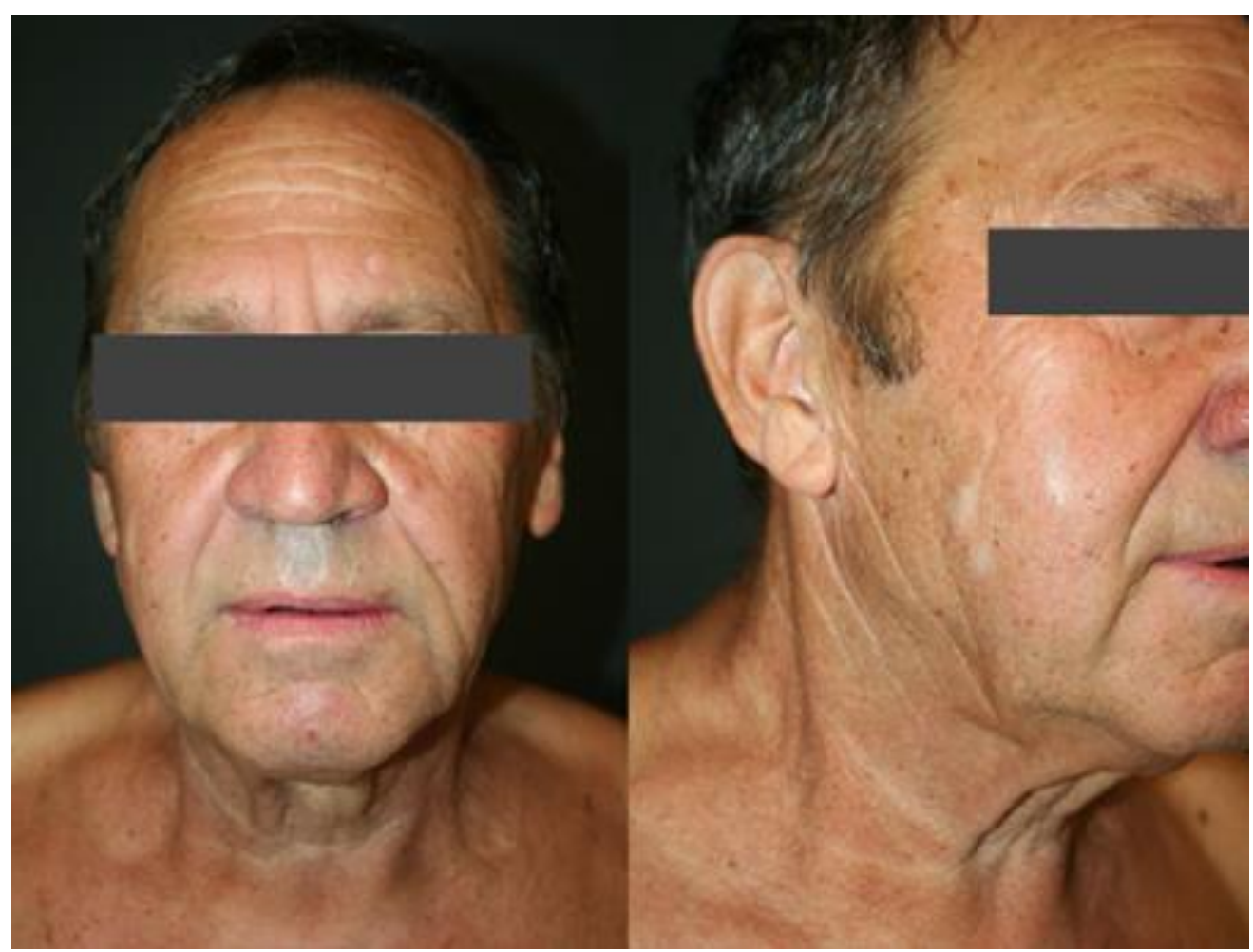

After the $\mathrm{CO} 2$ laser treatments, the patient discontinued treatment with Methotrexate. No lesion recurrence is observed. Further follow-up of the patient is ongoing.

\section{Discussion}

Systemic Rosai-Dorfman disease usually affects children or young adults, with a male predominance. Meanwhile cutaneous Rosai-Dorfman disease occurs at older age and has a female preponderance. Asian population is more frequently affected [3]. In our clinical case an older Caucasian man was diagnosed with cutaneous Rosai-Dorfman disease (CRDD) which is uncharacteristic to the illness. Patients with CRDD usually are healthy, without leading constitutional or other symptoms.

Etiology of this disorder is unclear. It is assumed to be a benign origin rather than a neoplastic, caused by infection or cellular immune infringement. [9] Any known association between Borrelia burgdorferi infection and any form of Rosai - Dorfman disease is not described before. Therefore it might be just a concomitant illness even though during the treatment with Amoxicillin lesions slowly diminished. In most cases lesions are asymptomatic nodules, plaques or papules, varying from erythematous to brown, grey-blue, violaceous or yellowish in color, usually slow growing [10]. Any location can be affected, extremities predominantly [11]. 
Diagnosis rests on distinctive histological features. The main finding is the presence of the characteristic foamy histiocytes. In addition, emperipolesis or lymphophagocytosis, the presence of intact inflammatory cells in the cytoplasm of histiocytes is common. On immunohistochemistry, histiocytes stain positive for S-100, CD4, factor XIIIa, either positive or negative for CD68 and negative for CD1a [12].

The majority of cases have a benign course and treatment is not necessary. Disease usually resolves spontaneously over a period of months to years [13]. Treatment should be administered to patients with life-threatening disease (which is not characteristic to disease limited only to the skin) or in non-responsive or multiple relapsing cases. Also, it is very important to take into consideration the patient's expectations and aesthetic appearance before making a treatment decision.

Various treatment options are described, including cryotherapy, radiotherapy, systemic, topical or intralesional corticosteroids, antibiotics, interferon, chemotherapy, methotrexate, dapsone [13] [14]. The most effective treatment option if the lesion is solitary is a surgical excision. On the other hand this treatment method is associated with a scar formation risk and lesion recurrence [15].

The treatment with $\mathrm{CO} 2$ laser has some advantages compared to other treatment options for its safe ablation (high efficacy in ablating benign raised lesions), good hemostasis, low cost and short recovery time [16]. The $\mathrm{CO} 2$ laser treatment was effective in our case. To our knowledge, this is only the second case where CRDD lesions were treated successfully with CO2 laser.

\section{Conclusion}

Rosai - Dorfman disease is a rare benign disorder of histiocytic proliferation usually affects younger people, but can occur at any age. Etiology of the disease is unclear. The majority of cases have a benign course and treatment is not necessary. Treatment should be administered to patients with life-threatening disease or in non-responsive or multiple relapsing cases. Also, it is very important to take into consideration the patient's wishes and aesthetic look before making a treatment decision.

\section{References}

[1] Rosai J, Dorfman RF. Sinus histiocytosis with massive lym- phadenopathy: a newly recognized benign clinicopathological entity. Arch Pathol 1969;87:63-70.

[2] Yoon AJ, Parisien M, Feldman F, et al. Extranodal rosai-dorfman disease of bone, subcutaneous tissue and paranasal sinus mucosa with a review of its pathogenesis. Skeletal Radiol 2005;34:6537.

[3] Lazar AP, Esterly NB, Gonzalez-Crussi F. Sinus histiocytosis clini- cally limited to the skin. Pediatr Dermatol 1987;4:247-53.

[4] Levine PH, Jahan N, Murari P, Manak M, Jaffe ES. Detection of human herpesvirus 6 in tissues involved by sinus histiocytosis with massive lymphadenopathy (Rosai-Dorfman disease). J Infect Dis 1992;166:291-5. 
[5] Abbas Al-Ramzia, Maysoun Kassema, Ashraf Barghasha, Karim M. Adel. A rare presentation of extra nodal rosai-dorfman disease (case report). Future Dental Journal Volume 3, Issue 1, June 2017, Pages 35-37.

[6] Brenn T, Calonje E, Granter SR, Leonard N, et al. Cutaneous rosai- dorfman disease is a distinct clinical entity. Am J Dermatopathol 2002; 24:385-91.

[7] Thawerani H, Sanchez RL, Rosai J. The cutaneous manifestations of sinus histiocytosis with massive lymphadenopathy. Arch Dermatol 1978;114:191-7.

[8] Al-Khateeb TH. Cutaneous Rosai-Dorfman disease of the face: a comprehensive litera- ture review and case report. J Oral Maxillofac Surg. 2016;74(3):528-540.

[9] Paulli M, Bergamaschi G, Tonon L, Viglio A, et al. Evidence for a polyclonal nature of the cell infiltrate in sinus histiocytosis with massive lymphadenopathy (Rosai-Dorfman disease). Br $\mathrm{J}$ Haematol 1995;91: 415-8.

[10] Abla O, Jacobsen E, Picarsic J, et al. Consensus recommendations for the diagnosis and clinical management of Rosai-Dorfman-Destombes disease. Blood 2018;131:2877-90.

[11] Kong Y-Y, Kong J-C, Shi D-R, et al. Cutaneous Rosai-Dorfman disease: a clinical and histopathologic study of 25 cases in China. Am J Surg Pathol 2007;31:341-50.

[12] Dalia S, Sagatys E, Sokol L, et al. Rosai-Dorfman disease: tumor biology, clinical features, pathology, and treatment. Cancer Control 2014;21:322-7.

[13] Felipe Barbosa Lima, Pedro Samuel de Valões Barcelos, Ana Paula Nunes Constâncio, Cleto Dantas Nogueira, and Antônio Aldo Melo-Filho. Rosai-Dorfman disease with spontaneous resolution: case report of a child. Rev Bras Hematol Hemoter. 2011; 33(4): 312-314.

[14] R.C. Maia1, E. de Meis, S. Romano, J.A. Dobbin, C.E. Klumb. Rosai-Dorfman disease: a report of eight cases in a tertiary care center and a review of the literature. Brazilian Journal of Medical and Biological Research. Braz J Med Biol Res vol.48 no.1 Ribeirão Preto Jan. 2015. Epub Oct 10, 2014.

[15] Al-Khateeb TH. Cutaneous rosai-dorfman disease of the face: a comprehensive literature review and case report. J Oral Maxillofac Surg 2016;74:528-40.

[16] Omi T, Numano $\mathrm{K}$. The Role of the $\mathrm{CO} 2$ Laser and Fractional $\mathrm{CO} 2$ Laser in Dermatology. Laser Ther. 2014 Mar 27;23(1):49-60. doi: 10.5978/islsm.14-RE-01. 